\title{
A smoothed-particle hydrodynamics model for ice-sheet and ice-shelf dynamics
}

\author{
Wenxiao PAN, ${ }^{1}$ Alexandre M. TARTAKOVSKY, ${ }^{1}$ Joe J. MONAGHAN ${ }^{2}$ \\ ${ }^{1}$ Pacific Northwest National Laboratory, Richland, WA, USA \\ E-mail: alexandre.tartakovsky@pnnl.gov \\ ${ }^{2}$ School of Mathematical Sciences, Monash University, Victoria, Australia
}

\begin{abstract}
Mathematical modeling of ice sheets is complicated by the nonlinearity of the governing equations and boundary conditions. Standard grid-based methods require complex front-tracking techniques and have a limited capability to handle large material deformations and abrupt changes in bottom topography. Consequently, numerical methods are usually restricted to shallow ice-sheet and ice-shelf approximations. We propose a new smoothed-particle hydrodynamics (SPH) model for coupled ice-sheet and ice-shelf dynamics. SPH, a fully Lagrangian particle method, is highly scalable and its Lagrangian nature and meshless discretization are well suited to the simulation of free surface flows, large material deformation and material fragmentation. In this paper, we use the SPH model to study ice-sheet/ice-shelf behavior, and the dynamics of the grounding line. The steady-state position of the grounding line obtained from SPH simulations is in good agreement with laboratory observations for a wide range of simulated bedrock slopes and density ratios, similar to those of ice and sea water. The numerical accuracy of the SPH algorithm is verified by simulating the plane-shear flow of two immiscible fluids and the propagation of a highly viscous blob of fluid along a horizontal surface. In the experiment, the ice was represented with a viscous Newtonian fluid. For consistency, in the described SPH model the ice is also modeled as a viscous Newtonian fluid. Typically, ice sheets are modeled as a non-Newtonian fluid, accounting for the changes in the mechanical properties of the ice. Implementation of a nonNewtonian rheology in the SPH model is the subject of our ongoing research.
\end{abstract}

\section{INTRODUCTION}

Most existing ice-sheet models use grid-based Eulerian discretizations and are based on various approximations to a steady-state Stokes equation (Marshall, 2005). The approximations are associated with the shallowness, namely the small depth-to-width ratio, of ice sheets to simplify the equations and reduce computational cost (Bueler and Brown, 2009). The simplest models use the first-order shallow-ice approximation (Morland and Johnson, 1980) and the shallow-shelf approximation (Weis and others, 1999). Under certain conditions, shallow-ice and shallow-shelf approximations may lead to significant errors. Examples of this include: large ice-sheet aspect ratio and/or large bedrock slope; tidewater glaciers; ice streams; surge dynamics; the dynamics of flow across the grounding line; and the dynamics in the vicinity of ice-sheet divides (Marshall, 2005). Recently, several models based on the Stokes equation or its high-order approximations have been proposed (Bueler and Brown, 2009; Durand and others, 2009; Pollard and DeConto, 2009; Price and others, 2011; Seddik and others, 2011; Seroussi and others, 2011), but the presence of the free surface (ice/air interface) still remains a major computational challenge for the Eulerian grid-based methods.

We propose a new smoothed-particle hydrodynamics (SPH) model for coupled ice-sheet and ice-shelf dynamics. $\mathrm{SPH}$ is a fully Lagrangian method that uses meshless (particle) discretization of the computational domain (Monaghan, 2005). SPH particles are used as discretization points for solving conservation equations. In the proposed SPH model, we solve the full momentum conservation equation, coupled with the continuity equation subject to the free surface boundary condition at the ice/water interface and the no-slip boundary condition at the ice/rock interface. Continuity of velocity and the viscous stress are imposed at the ice/water interface. Lagrangian particle methods do not require interface-tracking algorithms for modeling freesurface (e.g. Monaghan, 1994; Tartakovsky and Meakin, 2005) and moving boundary (multiphase) problems (e.g. Colagrossi and Landrini, 2003; Tartakovsky and others, 2007, 2009). In SPH, each fluid has its own set of particles and there is no need to define the interface. This makes SPH very efficient for ice-sheet modeling.

The marine ice sheets terminate in the ocean and exhibit a balance between snowfall inland and calving of icebergs into the ocean. Ice sheets thin as they flow toward the ocean and eventually detach from the bedrock to form freely floating ice shelves. The location of the detachment is called the grounding line. Recently, a laboratory experiment was performed by Robison and others (2010) to explore the dynamics of the grounding line between marine ice sheets and the freely floating ice shelves into which they develop. In this experiment, the ice sheets and shelves were represented by a viscous Newtonian fluid flowing down a ramp into a tank containing a denser and much less viscous fluid. The viscous fluid floated off and formed a shelf at the grounding line, which proceeded steadily (without retreating) and eventually reached a steady position. The experiment captures the fundamental dynamical properties of flow across a grounding line between a horizontal sheardominated ice sheet and an extensional deviatoric sheardominated ice shelf. We use the SPH model to simulate processes studied in the experiment, and we find excellent agreement between the steady positions of the grounding line obtained numerically and experimentally for a wide range of 
ramp (bedrock) slopes, density ratios and ice-sheet flow rates. Numerical results also agree with theoretical prediction for the location of the grounding line obtained by Robison and others (2010).

In order to obtain a consistent comparison with the experimental data, in the SPH model the ice sheet and ice shelf are modeled as a viscous Newtonian fluid. In reality, ice sheets have a complex rheology and are traditionally modeled as a non-Newtonian fluid with rheology represented by Glen's power law (Huybrechts and others, 1996; Goldsby and Kohlsted, 2001). However, the rheology of the ice sheet and ice shelf primarily affects the vertical velocity profiles within them, and the effect of the rheology on the depth-averaged quantities and position of the grounding line is unknown. Implementation of the non-Newtonian rheology in the SPH method is relatively straightforward (Shao and Lo, 2003; Hosseini and others, 2007). The effect of the non-Newtonian rheological properties of ice on the ice-sheet dynamics is a subject of our ongoing research.

This paper is organized as follows. First we describe the SPH model. Next, the model is verified against finitedifference and analytical solutions for the plane-shear flow of two immiscible fluids and the propagation of gravity current along a rigid solid surface. Finally, we compare the dynamics of the grounding line obtained from $\mathrm{SPH}$ simulations with the laboratory experiment of Robison and others (2010).

\section{SPH DISCRETIZATION OF THE NAVIER-STOKES AND CONTINUITY EQUATIONS \\ SPH equations of motion}

In the proposed model, the SPH method is used to discretize the governing Navier-Stokes and continuity equations. SPH uses a meshless (particle) discretization of the computational domain (see Monaghan, 2005, for a review). The SPH interpolation scheme, $A(\mathbf{r})=\sum_{i} \frac{m_{i}}{\rho_{i}} A_{i} W\left(\mathbf{r}-\mathbf{r}_{i}, h\right)$, allows approximation of a continuous field, $A(\mathbf{r})$, using the values of $A$ at a set of discretization points. Here $\mathbf{r}_{i}$ is the position of discretization point $i, A_{i}=A\left(\mathbf{r}_{i}\right)$, and $\rho_{i}$ and $m_{i}$ are the density and the mass of the phase associated with point $i$. Because each point possesses a mass and volume, $m_{i} / \rho_{i}$, it is natural to think of discretization points as physical particles. $W$ is the bell-shaped SPH weighting function with compact support $k h\left(W\left(\left|\mathbf{r}-\mathbf{r}_{i}\right|>k h, h\right)=0\right)$, where $|\mathbf{r}|$ is the magnitude of a vector $\mathbf{r}$, the value of $k$ depends on a functional form of $W$ and $h$ depends on position $\mathbf{r}$. The interpolation scheme assumes summation over all the SPH particles within a distance $k h$ of $\mathbf{r}$. We choose a cubic spline kernel, which is defined as

$$
W(\mathbf{r}, h)=\frac{a_{D}}{h^{D}} \begin{cases}1-\frac{3}{2} x^{2}+\frac{3}{4} x^{3} & 0 \leq x \leq 1 \\ \frac{1}{4}(2-x)^{3} & 1<x \leq 2 \\ 0 & x>2\end{cases}
$$

where $x=|\mathbf{r}| / h, D$ is the spatial dimension and $a_{D}$ is a constant that assures proper normalization of the smoothing function $\left(a_{D}=2 / 3,10 / 7 \pi\right.$ and $1 / \pi$ for $D=1,2$ and 3 , respectively). Here only particles within $2 h$ of the central particle contribute to the smoothing kernel, which is with continuous second derivatives for all $r$. The gradient of the kernel is

$$
\nabla W(\mathbf{r}, h)=\nabla|\mathbf{r}| \frac{a_{D}}{h^{D+1}} \begin{cases}\frac{9}{4} x^{2}-3 x & 0 \leq x \leq 1 \\ -\frac{3}{4}(2-x)^{2} & 1<x \leq 2 \\ 0 & x>2\end{cases}
$$

The SPH approximation of continuous fields and their spatial derivatives allow the continuity and Navier-Stokes equations to be written in the form of a system of ordinary differential equations (Monaghan, 2005):

$$
\begin{gathered}
\frac{\mathrm{d} \rho_{i}}{\mathrm{~d} t}=\rho_{i} \sum_{j} \frac{m_{j}}{\rho_{j}} \mathbf{v}_{i j} \cdot \nabla_{i} W\left(\mathbf{r}_{i j}, \bar{h}\right) \\
\frac{\mathrm{d} \mathbf{v}_{i}}{\mathrm{~d} t}=-\sum_{j} m_{j}\left(\frac{P_{i}+P_{j}}{\rho_{i} \rho_{j}}+0.08\left|\frac{P_{i}+P_{j}}{\rho_{i} \rho_{j}}\right|\left|\frac{\rho_{0}^{1}-\rho_{0}^{2}}{\rho_{0}^{1}+\rho_{0}^{2}}\right|+\Pi_{i j}\right) \\
\times \nabla_{i} W\left(\mathbf{r}_{i j}, \bar{h}\right)+\mathbf{g}
\end{gathered}
$$

where

$$
\Pi_{i j}=-\frac{112}{15} \frac{\mathbf{v}_{i j} \cdot \mathbf{r}_{i j}}{\left|\mathbf{r}_{i j}\right|} \frac{2 \mu_{i} \mu_{j}}{\left(\mu_{i}+\mu_{j}\right) h \rho_{i} \rho_{j}},
$$

$\mathbf{r}_{i j}=\mathbf{r}_{i}-\mathbf{r}_{j}, \mathbf{v}_{i j}=\mathbf{v}_{i}-\mathbf{v}_{j}, \bar{h}=\left(h_{i}+h_{j}\right) / 2, P_{i}$ is the fluid pressure and $\mu_{i}$ is the fluid viscosity at position $\mathbf{r}_{i} ; \rho_{0}^{1}$ and $\rho_{0}^{2}$ represent the reference densities of fluids 1 and 2 , respectively. In Eqn (4), the first term inside the summation is a repulsive force acting between particles $i$ and $j$ that is obtained from the discretization of the pressure gradient term in the Navier-Stokes equation. The second term is a repulsive force that is needed only if the density difference between two fluids is large (e.g. a density ratio of 5 to 10 or more (Monaghan, 1994)). The third term is the viscous force defined in Eqn (5). The attractive feature of Eqn (4) is that the forces have antisymmetric form with respect to indexes $i$ and $j$ (forces acting between the SPH particles satisfy Newton's third law). Because of this, Eqn (4) satisfies the linear and angular momentum conservation laws exactly.

Ice, water, bedrock and the ocean bottom are represented by different sets of particles with different masses, viscosities and densities. In Eqns (3) and (4), $\sum_{j}$ indicates summation over all neighboring particles of particle $i$. Particles representing solid walls are fixed in space. They enter into the calculation of the densities of fluid particles (Eqn (3)) and forces acting on the fluid particles (Eqn (4)). $\mathbf{v}$ is the fluid velocity vector, $P$ is the pressure, $\mathbf{g}$ is the gravitational acceleration vector and $\Pi$ is the artificial viscosity.

For computational efficiency, we approximate an incompressible fluid as a slightly compressible fluid. The equation of state, $P_{i}=P_{0}\left[\left(\rho_{i} / \rho_{0}\right)^{7}-1\right]$, was used to close the system of Eqns (3) and (4). In the equations of state, $\rho_{0}$ is the reference density, and $P_{0}$ is the magnitude of pressure given by $7 P_{0} / \rho_{0}=25 V_{\max }^{2}=c_{i}^{2}$. Here $c_{i}$ is the speed of sound of particle $i$. This equation of state limits the relative density fluctuation to $V_{\max } / c_{i}^{2}=1 / 25$. Under such conditions, the $\mathrm{SPH}$ fluids behave like incompressible fluids.

One layer of solid (boundary) particles is placed along the fluid/solid boundary, and forces acting between the boundary particles and fluid particles near the boundary are added to Eqn (4) to ensure that the normal velocity is zero (Monaghan and Kajtar, 2009). To satisfy the no-slip boundary condition, the boundary particles are included in the calculation of viscous forces acting on the fluid particles. The boundary force is calculated as

$$
\mathbf{F}_{i}^{\mathrm{b}}=\sum_{j} V_{\max }^{2} \frac{2 m_{j}}{m_{i}+m_{j}} W^{\mathrm{b}}\left(\mathbf{r}_{i j}, \bar{h}\right) \frac{\mathbf{r}_{i j}}{\left|\mathbf{r}_{i j}\right|} \frac{1}{\left|\mathbf{r}_{i j}\right|-\Delta p_{\mathrm{b}}},
$$


Table 1. Parameters of the two fluids

\begin{tabular}{llc}
\hline Fluid & $\begin{array}{c}\nu=\mu / \rho \\
\mathrm{m}^{2} \mathrm{~s}^{-1}\end{array}$ & $\begin{array}{c}\rho \\
\mathrm{kg} \mathrm{m}^{-3}\end{array}$ \\
\hline 1 & 0.001 & 1500 \\
2 & 0.01 & 1300 \\
\hline
\end{tabular}

where particle $j$ is the neighbor boundary particle of fluid particle $i, W^{\mathrm{b}}(\mathbf{r}, h)=\frac{1}{8}\left(1+\frac{3}{2} \frac{|\mathbf{r}|}{h}\right)\left(2-\frac{|\mathbf{r}|}{h}\right)^{3}$ for $|\mathbf{r}| / h \leq 2$ is proportional to a Wendland one-dimensional (1-D) cubic kernel. The boundary particle spacing, $\Delta p_{\mathrm{b}}$, is less than the fluid particle spacing by a factor of 2 or more. Monaghan and Kajtar (2009) demonstrated that for $\Delta p_{\mathrm{b}}$ satisfying this condition, the total boundary force on a fluid SPH particle acts in the direction perpendicular to the boundary within a negligible error.

Finally, the smoothing length, $h$, determines the resolution and the number of neighbors that contribute to the properties at a point. The efficiency and the accuracy would therefore be greater if $h$ was chosen so that it depended on the local particle number density. Typically, $h_{i}$ is calculated from

$$
\frac{\mathrm{d} h_{i}}{\mathrm{~d} t}=-\left(\frac{h_{i}}{D \rho_{i}}\right) \frac{\mathrm{d} \rho_{i}}{\mathrm{~d} t},
$$

where $D$ is the number of dimensions (Monaghan, 1992). A symmetric kernel is obtained by replacing $h$ with the arithmetic mean of the smoothing lengths for the two particles as $h=\left(h_{i}+h_{j}\right) / 2$ (Monaghan, 1992).

\section{Integration and time-stepping}

The second-order leapfrog integrator is used to integrate the $\mathrm{SPH}$ equations of motion. For known position, $\mathbf{r}$, velocity, $\mathbf{v}$, and acceleration, $\mathbf{f}$, at time $t$, the leapfrog integrator gives the positions and velocities at time $t+\Delta t$ as

$$
\begin{aligned}
& \mathbf{r}_{i}\left(t+\frac{\Delta t}{2}\right)=\mathbf{r}_{i}(t)+\frac{\Delta t}{2} \mathbf{v}_{i}(t) \\
& \mathbf{v}_{i}\left(t+\frac{\Delta t}{2}\right)=\mathbf{v}_{i}(t)+\frac{\Delta t}{2} \mathbf{f}_{i}\left(t-\frac{\Delta t}{2}\right) \\
& \mathbf{v}_{i}(t+\Delta t)=\mathbf{v}_{i}(t)+\Delta t \mathbf{f}_{i}\left(t+\frac{\Delta t}{2}\right) \\
& \mathbf{r}_{i}(t+\Delta t)=\mathbf{r}_{i}\left(t+\frac{\Delta t}{2}\right)+\frac{\Delta t}{2} \mathbf{v}_{i}(t+\Delta t) .
\end{aligned}
$$

The smoothing length, $h_{i}(t)$, and density, $\rho_{i}(t)$, are integrated as

$$
\begin{aligned}
& \rho_{i}\left(t+\frac{\Delta t}{2}\right)=\rho_{i}(t)+\frac{\Delta t}{2} R_{i}(t) \\
& h_{i}\left(t+\frac{\Delta t}{2}\right)=h_{i}(t)\left[1-\frac{1}{D \rho_{i}\left(t+\frac{\Delta t}{2}\right)} \frac{\Delta t}{2} R_{i}(t)\right] \\
& \rho_{i}(t+\Delta t)=\rho_{i}\left(t+\frac{\Delta t}{2}\right)+\frac{\Delta t}{2} R_{i}(t) \\
& h_{i}(t+\Delta t)=h_{i}\left(t+\frac{\Delta t}{2}\right) /\left[1+\frac{1}{D \rho_{i}(t+\Delta t)} \frac{\Delta t}{2} R_{i}(t)\right],
\end{aligned}
$$

where $R_{i}(t)=\mathrm{d} \rho_{i}(t) / \mathrm{d} t$.

With this integrator, it is crucial that the time-step size is chosen correctly, both to ensure the accuracy of the evolution and to ensure numerical stability. The time-step is chosen as the minimum of the Courant-Friedrichs-Lewy condition,

$$
\Delta t \leq \min _{i j} \frac{2 h}{C_{i}+c_{j}},
$$

the viscosity condition (Cleary and Monaghan, 1999),

$$
\Delta t \leq 0.1 \min _{i j} \frac{h^{2}\left(\rho_{i}+\rho_{j}\right)\left(\mu_{i}+\mu_{j}\right)}{4 \mu_{i} \mu_{j}},
$$

and the boundary force condition (Monaghan and Kajtar, 2009),

$$
\Delta t \leq \min _{i j} \frac{\left|\mathbf{r}_{i j}-\Delta p_{\mathrm{b}}\right|}{V_{\mathrm{b}}}
$$

where $V_{\mathrm{b}}=\max \left(\left|\mathbf{v}_{i j}\right|, V_{\max }\right)$.

\section{NUMERICAL RESULTS}

To verify our SPH algorithm we applied two tests: (1) the plane-shear flow of two fluids with different densities and viscosities, a fixed, no-slip, horizontal bottom boundary and an upper free surface; and (2) the evolution of a blob of viscous fluid spreading along a horizontal boundary.

\section{Plane-shear flow of two fluids}

We study a two-dimensional (2-D) plane-shear flow of two immiscible fluids with a free surface. A layer of fluid 1 is bounded by an impermeable plane boundary at the bottom moving with velocity $u_{0}$ and a layer of fluid 2 at the top. The shear flow is initiated by a sudden stop of the bottom plane. For this problem, the (2-D) Navier-Stokes and continuity equations can be reduced to $1-\mathrm{D}$ equations:

$$
\begin{array}{cc}
\rho_{1} \frac{\partial u(t, y)}{\partial t}=\mu_{1} \frac{\partial^{2} u(t, y)}{\partial y^{2}} & 0<y<0.5 \\
\rho_{2} \frac{\partial u(t, y)}{\partial t}=\mu_{2} \frac{\partial^{2} u(t, y)}{\partial y^{2}} & 0.5<y<1,
\end{array}
$$

subject to initial and boundary conditions:

$$
\left\{\begin{array}{lll}
u(t, y)=u_{0} & \text { at } \quad 0 \leq y \leq 1 & t=0 \\
u(t, y)=0 & \text { at } y=0 & t>0 \\
\frac{\partial u}{\partial y}=0 & \text { at } y=1 & t>0 \\
\mu_{1} \frac{\partial u}{\partial y}=\mu_{2} \frac{\partial u}{\partial y} & \text { at } y=\frac{1}{2} & t>0
\end{array}\right.
$$

The parameters of the two fluids are given in Table 1. We use the SPH model to solve the 2-D continuity and Navier-Stokes equations and use finite-difference (FD) solutions of Eqns (13) and (14) to verify the SPH solution. The FD scheme has firstorder accuracy in time and second-order accuracy in space and has the grid size $\Delta y=0.005$. In the SPH simulations, pressure and velocity are assumed to be periodic in the $x$ direction. The computational domain in the $x$-direction is set to $10 \mathrm{~h}$ (to avoid the effect of the periodic boundary condition), and SPH particles exiting domain at $x=10 \mathrm{~h}$ with velocity $\mathbf{v}_{i}$ are returned in the domain at $x=0$ with the same velocity. Figure 1 compares an SPH solution of the continuity and Navier-Stokes equations, obtained with different resolution, $\Delta p$, with a FD solution of the equivalent 1-D Eqns (13) and (14) for three different times. In the $\mathrm{SPH}$ solution, $\Delta p$ is the initial spacing between the SPH particles. The figure shows that SPH solutions approach the FD solution with decreasing $\Delta p$ (increasing resolution of the SPH solution). Figure 2 shows the $L_{2}$ relative error versus $\Delta p$ at three times. At $t=40.7 \mathrm{~s}$, the error is found to decrease as $\Delta p^{1.0}$, while at $t=309.3 \mathrm{~s}$ it decreases as $\Delta p^{1.5}$, and at $t=773.2 \mathrm{~s}$ the error decreases as $\Delta p^{0.6}$. We find that the shear free surface flow is simulated accurately by the SPH model and the accuracy is improved with increasing spatial resolution.

\section{Gravity current along a rigid surface}

Next, we study a viscous gravity current propagating over a rigid horizontal surface, a problem that is often used to verify ice-sheet codes (Bueler and others, 2007). This test imitates 


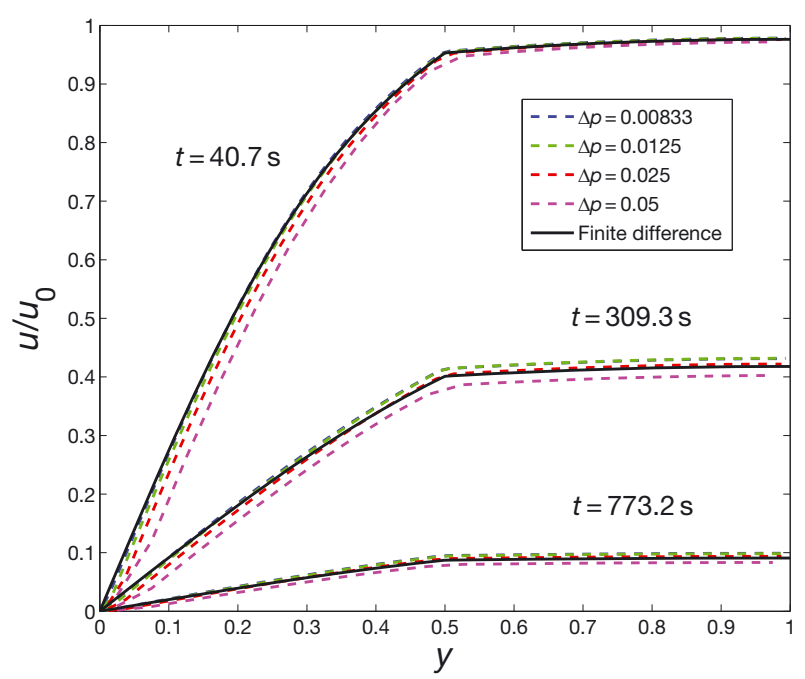

Fig. 1. The velocity profiles of the flow at different times.

a volume of ice that spreads as a sheet over a horizontal surface under gravity. In the absence of surface tension, this problem allows a 2-D analytical solution (Huppert, 1982). We simulate the gravity current propagation with the SPH model in two dimensions and use the analytical solution to verify the SPH model.

The analytical solution is obtained using the lubricationtheory approximation as follows. A current of density $\rho$ is considered to be propagating over a rigid horizontal surface with depth $H(x, t)$. Here the vertical velocities are negligibly small and the pressure is hydrostatic. The balance between the pressure gradient and the viscous forces then gives the governing nonlinear partial differential equation for $H(x, t)$ (Huppert, 1982):

$$
\frac{\partial H}{\partial t}-\frac{1}{3} \frac{g}{\nu} \frac{\partial}{\partial x}\left(H^{3} \frac{\partial H}{\partial x}\right)=0,
$$

where $\nu$ is the kinematic viscosity of the viscous fluid. For an incompressible fluid the continuity equation yields

$$
\int_{0}^{L} H(x, t) \mathrm{d} x \equiv q
$$

where $q$ is the constant volume of the current. A similarity solution of $H(x, t)$ can be found in terms of the similarity variable (Huppert, 1982),

$$
\eta=\left(\frac{1}{3} g q^{3} / \nu\right)^{1 / 5} x t^{-1 / 5}
$$

and the solution is then expressed as (Huppert, 1982)

$$
H(x, t)=\eta_{L}^{2 / 3}\left(3 q^{2} \nu / g\right)^{1 / 5} t^{-1 / 5} \phi\left(\eta / \eta_{L}\right),
$$

where $\eta_{L}$ is the value of $\eta$ at $x=L(t)$, and $L(t)$ satisfies $H(L, t) \equiv 0$. The function $\phi(y)$ with $y=\eta / \eta_{L}$ satisfies

$$
\left(\phi^{3} \phi^{\prime}\right)^{\prime}+\frac{1}{5} y \phi^{\prime}+\frac{1}{5} \phi=0
$$

and

$$
\eta_{L}=\left(\int_{0}^{1} \phi(y) d y\right)^{-3 / 5}
$$

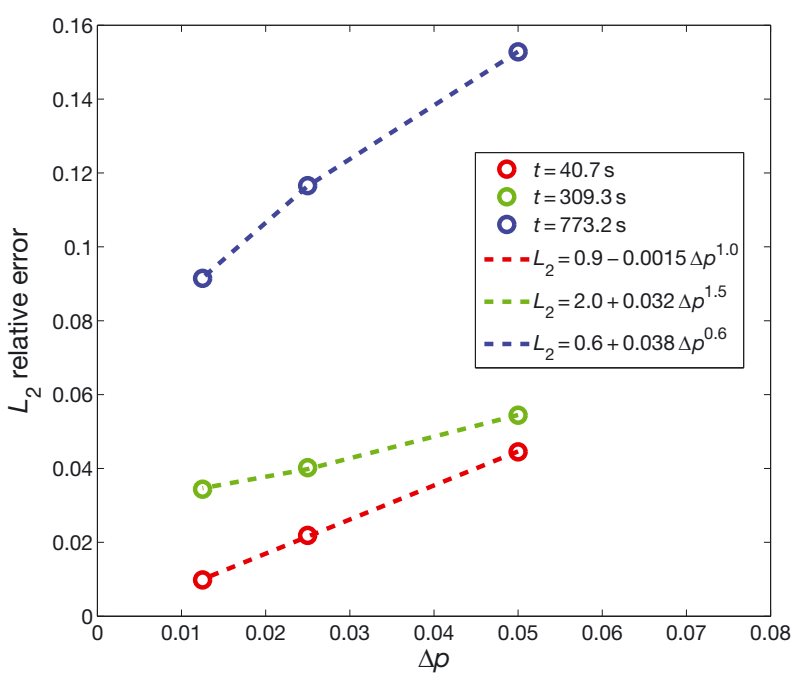

Fig. 2. The $L_{2}$ relative error of the velocity.

The analytical solution of Eqns (19) and (20) is obtained as:

$$
\phi(y)=\left(\frac{3}{10}\right)^{1 / 3}\left(1-y^{2}\right)^{1 / 3} \text { and } \eta_{L}=1.411 \cdots
$$

Figure 3 compares the ice-sheet thickness over time, $H(x, t)$, obtained from the SPH simulation and the analytical solution at different times. Figure 4 shows the margin length, $L(t)$, as function of time obtained analytically, and from the SPH simulations with different resolutions. These figures demonstrate that the SPH solution converges to the analytical solution with decreasing $\Delta p$ (increasing resolution).

\section{Dynamics of the grounding line}

To demonstrate the capabilities of the SPH ice-sheet model, we use the model to investigate the dynamics of the grounding line, and compare it with the laboratory observations of Robison and others (2010) and their theoretical prediction.

In Robison and others' (2010) laboratory experiment, a Newtonian viscous fluid (golden syrup) was supplied from a reservoir onto a ramp, down which it flowed into a tank filled with aqueous potassium carbonate solution. The syrup is more viscous but less dense than potassium carbonate solution and is able to float on the top of the 'ocean'. A constant head of golden syrup was maintained in the supply chamber. The flux rate was adjusted by altering the head height. At a certain point (the grounding line) along the ramp, the syrup floated off and formed a shelf. A side-view camera was used to record and measure the positions of the

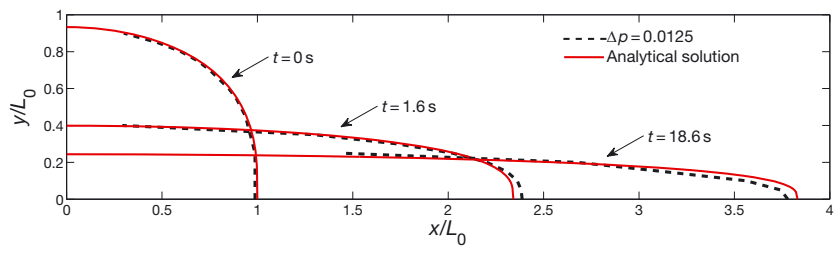

Fig. 3. The surface profile of the ice sheet over time as the sheet spreads under gravity. The dashed curves represent the solution of Huppert (1982). Here, $L_{0}$ is the initial margin length and $\Delta p$ is the initial particle spacing. 


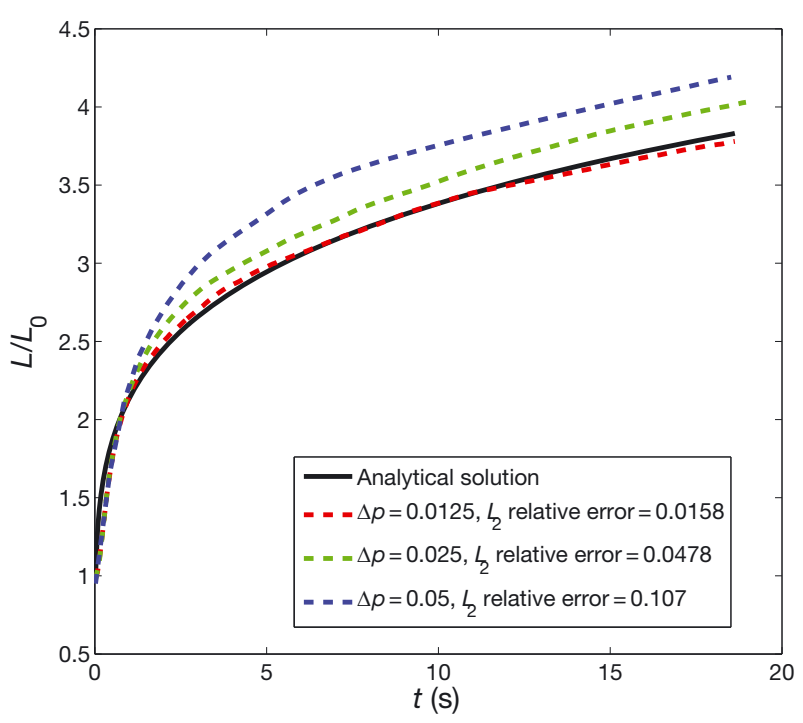

Fig. 4. Length of the ice sheet as a function of time obtained analytically and from SPH simulations with different initial particle spacing, $\Delta p$.

grounding line. The grounding line was found to move down the ramp but eventually reach a steady position.

By approximating the flow of an ice sheet as sheardominated and the flow of ice shelves as extensional with zero shear to leading order, Robison and others (2010) obtained an approximate analytical solution for the steady position of the grounding line,

$$
x_{\mathrm{G}}=(A+\epsilon)^{-1},
$$

where the dimensionless grounding line

$$
x_{\mathrm{G}}=2 B C^{4} x
$$

and

$$
\begin{aligned}
& A=2 \alpha\left(g^{\prime} / g\right)^{-1 / 2}, \quad B=\left(6 \nu q_{0} / g\right)^{1 / 3}, \\
& C=\left(g / g^{\prime}\right)^{1 / 6}, \quad \epsilon=2 \alpha\left(g^{\prime} / g\right)^{1 / 2}\left(\rho_{\mathrm{w}} / \rho\right) .
\end{aligned}
$$

Here $\alpha$ is the bed slope, $g$ is the gravity acceleration, $g^{\prime}=g\left(\rho_{\mathrm{w}}-\rho\right) / \rho_{\mathrm{w}}$ is the reduced gravity and $\rho$ and $\nu$ are the density and kinematic viscosity of the viscous fluid, respectively; $\rho_{\mathrm{w}}$ is the density of the dense fluid and $q_{0}$ is the flux of viscous fluid.

The set-up of a typical simulation is illustrated in Figure 5, which has the same dimensions as the laboratory set-up of Robison and others (2010). Viscous light (gray) fluid flows down a rigid ramp with slope $\alpha$ into the denser but less viscous 'ocean' (blue). The gray fluid is supplied through a

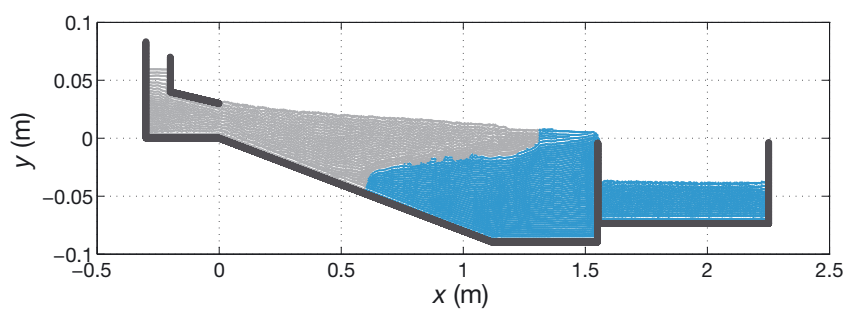

Fig. 5. The set-up used in our simulation. A viscous light fluid (gray) flows down onto a ramp and into a dense fluid (blue).

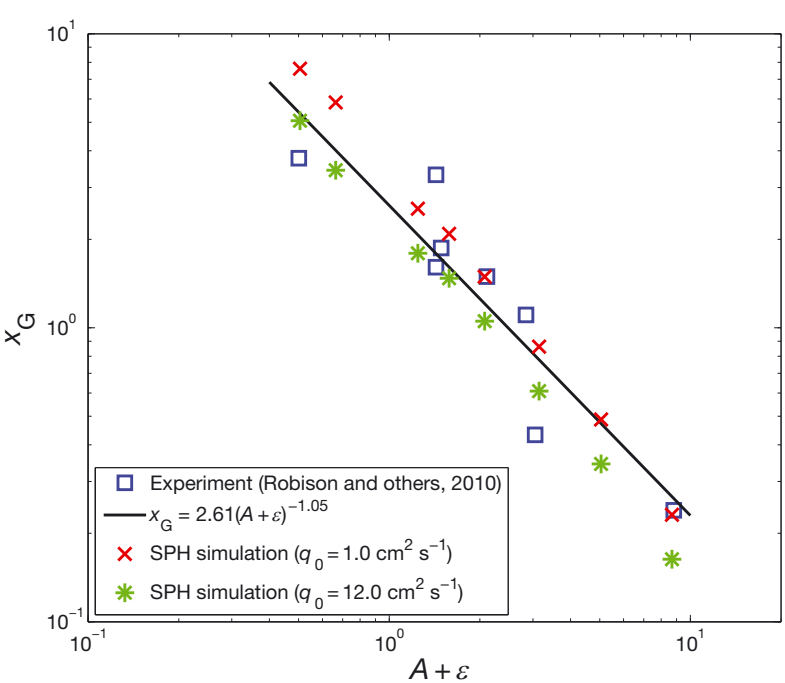

Fig. 6. The steady positions of the grounding line calculated in our simulations, compared with those measured in the experiment of Robison and others (2010). Different densities, $\rho$, and bed slopes, $\alpha$, were chosen according to the values used in the experiment (Table 2) for varied values of $A+\epsilon . x_{\mathrm{G}}$ is dimensionless, as defined in Eqn (23).

funnel from a reservoir where a constant level of fluid is maintained. An overflow tank is attached on the right to keep the constant 'sea level'.

To initialize a simulation, the particles were placed on the vertices of a grid of squares with lattice size $\Delta p$. Gray particles were placed inside the reservoir and the funnel, and blue particles were placed inside the basin. The overflow tank was initially empty. One layer of 'boundary' particles was placed along the solid boundary (the dark gray line) with spacing $\Delta p_{\mathrm{b}}=\Delta p / 3$. At time $t=0$, gravity drove the fluid particles (gray) to flow down the ramp and into the basin of blue particles. The flux of gray particles was controlled with the friction force, $-k \mathbf{v}_{\mathbf{i}}$, added into the momentum conservation, Eqn (4), for all particles within the funnel. The constant level of viscous fluid in the reservoir was maintained by inserting gray particles whenever the level drop exceeded $\Delta p$. The number of particles in the simulations ranged from 15000 to 20000, and the CPU time was $\sim 4$ hours on a standard single-processor desktop computer. The typical time-step used in the simulations was $\Delta t=0.0001 \mathrm{~s}$.

Figure 6 compares the steady position of the grounding line obtained from the SPH simulations and measured in the experiment (Robison and others, 2010). The good overall comparison between numerical and experimental results indicates that the SPH model accurately captures the position of the contact line for a wide range of slopes, fluxes and density ratios.

Table 2. Values used for the input parameters in the grounding line simulations

\begin{tabular}{lcccc}
\hline$\alpha$ & $\begin{array}{c}\nu \\
\mathrm{m}^{2} \mathrm{~s}^{-1}\end{array}$ & $\begin{array}{c}\nu_{\mathrm{w}} \\
\mathrm{m}^{2} \mathrm{~s}^{-1}\end{array}$ & $\begin{array}{c}\rho \\
\mathrm{kg} \mathrm{m}^{-3}\end{array}$ & $\begin{array}{l}\rho_{\mathrm{w}} \\
\mathrm{kg} \mathrm{m}^{-3}\end{array}$ \\
\hline $0.08-0.25$ & 0.01 & 0.001 & $1300-1495$ & 1500 \\
\hline
\end{tabular}


The convergence of the simulation was also examined. The position of the grounding line as a function of time was calculated at different spatial resolutions, $\Delta p$. In all the simulations, the position of the grounding line increased with time to an asymptotic value. Figure 7 shows that the asymptotic values (the steady-state position of the grounding line) converge with decreasing $\Delta p$. We find that a sufficiently accurate value of the steady position of the grounding line can be obtained with $\Delta p=0.026$, and we chose this resolution for all the simulations.

The black solid curve in Figure 6 is a linear fit to the experimental data, has a slope $-\beta=-1.05$ and follows a scaling law:

$$
x_{\mathrm{G}}=\gamma(A+\epsilon)^{-\beta} \text {. }
$$

The position of the grounding line, $x_{\mathrm{G}}$, obtained from the $\mathrm{SPH}$ simulations has a similar scaling behavior, with $\beta=1$.1. The slight discrepancy in the values of $\beta$ for experimental and numerical data and the significant scatter of the experimental data around the line given by Eqn (25) can be explained by several factors. The SPH simulations are 2-D, while the experiments are 3-D. In the experiment, the side-walls of the tank exert viscous shear stresses on the fluid, and, in some experiments, propagation of the syrup on the water surface was unstable (fingering of the syrup was observed). Both the experimental and numerical data show scaling behavior similar to the power law, Eqn (22), obtained theoretically by Robison and others (2010). However, the theory results in $\gamma=1$, while both the experimental and numerical results predict $\gamma \approx 3$. This discrepancy can be explained by the shallow-ice approximation used in the theoretical model.

\section{CONCLUSIONS}

A SPH model for ice-sheet dynamics was developed. The model was verified using analytical and numerical solutions for plane-shear flow of two immiscible fluids and the propagation of a gravity current along a rigid solid surface. The SPH simulations converge to exact solutions with sufficient spatial resolution. We have shown that the essential dynamics of marine ice sheets can be captured by SPH simulations. The SPH model was validated using data from an experiment of Robison and others (2010), mimicking the flow of an ice sheet and ice shelf. The experimental and numerical results show the migration of the grounding line between a grounded sheet and a freely floating shelf toward a steady position. The steady-state positions of the grounding line, obtained from the SPH simulations and the laboratory experiments, agree well for a wide range of bedrock slopes, density ratios and ice-sheet fluxes.

Typically, ice sheets are modeled as a non-Newtonian fluid (Huybrechts and others, 1996; Goldsby and Kohlsted, 2001), accounting for nonlinear dependence of the deviatoric stress on the strain rate. While in contact with bedrock, the flow of an ice sheet is resisted by shear stresses, both internal and at its base. However, beyond the grounding line, the ice shelf is characterized by a weak internal straining flow, with negligible tangential stress exerted upon it by the air above it or the ocean beneath. The effect of the non-Newtonian rheological properties of ice on the ice-sheet dynamics is the subject of our ongoing research.

Several important processes, such as basal melting/freezing, ice accumulation and basal sliding were absent in the experiments of Robison and others (2010). Including

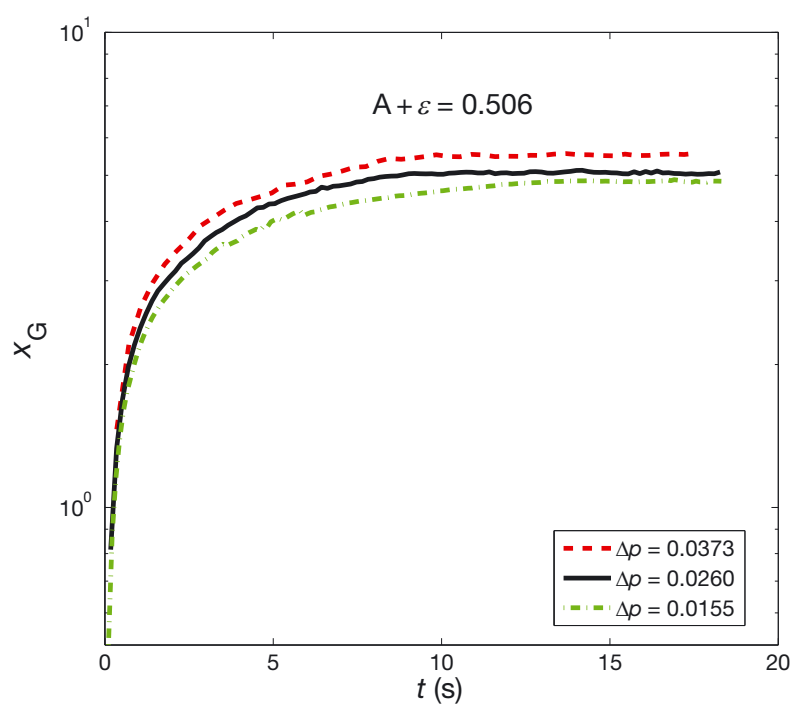

Fig. 7. The position of the grounding line as a function of time calculated at different spatial resolutions, $\Delta p$. All the variables are made dimensionless by Eqn (23).

these processes in the SPH ice-sheet model is the subject of our future research. An SPH framework for modeling melting/freezing processes was proposed by Monaghan and others (2005). The SPH deposition model for mineral growth of Tartakovsky and others $(2007,2008)$ can be used to model ice accumulation due to precipitation. Basal sliding in the SPH model can be modeled by prescribing the basal sliding velocity to the boundary particles. Implementation of a Robin boundary condition, which is commonly used in ice-sheet models to describe basal sliding (Gagliardini and others, 2007), in SPH is described by Ryan and others (2010).

\section{ACKNOWLEDGEMENTS}

This research was supported by the Scientific Discovery through Advanced Computing Program of the Office of Science, US Department of Energy. The Pacific Northwest National Laboratory is operated by Battelle for the US Department of Energy under contract DE-AC06-76RL01830.

\section{REFERENCES}

Bueler E and Brown J (2009) Shallow shelf approximation as a 'sliding law' in a thermomechanically coupled ice sheet model. J. Geophys. Res., 114(F3), F03008 (doi: 10.1029/2008JF001179)

Bueler E, Brown J and Lingle C (2007) Exact solutions to the thermomechanically coupled shallow-ice approximation: effective tools for verification. J. Glaciol., 53(182), 499-516

Cleary PW and Monaghan JJ (1999) Conduction modelling using smoothed particle hydrodynamics. J. Comput. Phys., 148(1), 227-264

Colagrossi A and Landrini M (2003) Numerical simulation of interfacial flows by smoothed particle hydrodynamics. J. Comput. Phys., 191(2), 448-475

Durand G, Gagliardini O, Zwinger T, Le Meur E and Hindmarsh RCA (2009) Full Stokes modeling of marine ice sheets: influence of the grid size. Ann. Glaciol., 50(52), 109-114

Gagliardini O, Cohen D, Råback P and Zwinger T (2007) Finiteelement modeling of subglacial cavities and related friction law. J. Geophys. Res., 112(F2), F02027 (doi: 10.1029/2006JF000576) 
Goldsby DL and Kohlsted DL (2001) Superplastic deformation of ice: experimental observations. J. Geophys. Res., 106(B6), $11017-11030$

Hosseini SM, Manzari MT and Hannani SK (2007) A fully explicit three-step SPH algorithm for simulation of non-Newtonian fluid flow. Int. J. Num. Meth. Heat Fluid Flow, 17(7), 715-735

Huppert HE (1982) The propagation of two-dimensional and axisymmetric viscous gravity currents over a rigid horizontal surface. J. Fluid Mech., 121, 43-58

Huybrechts P, Payne T and the EISMINT Intercomparison Group (1996) The EISMINT benchmarks for testing ice-sheet models. Ann. Glaciol., 23, 1-12

Marshall SJ (2005) Recent advances in understanding ice sheet dynamics. Earth Planet. Sci. Lett., 240(2), 191-204

Monaghan JJ (1992) Smoothed particle hydrodynamics. Annu. Rev. Astron. Astrophys., 30, 543-574

Monaghan JJ (1994) Simulating free surface flows with SPH. J. Comput. Phys., 110(2), 399-406

Monaghan JJ (2005) Smoothed particle hydrodynamics. Rep. Prog. Phys., 68(8), 1703-1759

Monaghan JJ and Kajtar JB (2009) SPH particle boundary forces for arbitrary boundaries. Comput. Phys. Commun., 180(10), $1811-1820$

Monaghan JJ, Huppert HE and Worster MG (2005) Solidification using smoothed particle hydrodynamics. J. Comput. Phys., 206(2), 684-705

Morland LW and Johnson IR (1980) Steady motion of ice sheets. J. Glaciol., 25(92), 229-246

Pollard D and DeConto RM (2009) Modelling West Antarctic ice sheet growth and collapse through the past five million years. Nature, 458(7236), 329-332

Price SF, Payne AJ, Howat IM and Smith BE (2011) Committed sea-level rise for the next century from Greenland ice sheet dynamics during the past decade. Proc. Natl Acad. Sci. USA (PNAS), 108(22), 8978-8983

Robison RAV, Huppert HE and Worster MG (2010) Dynamics of viscous grounding lines. J. Fluid Mech., 648, 363-380

Ryan EM, Tartakovsky AM and Amon C (2010) A novel method for modeling Neumann and Robin boundary conditions in smoothed particle hydrodynamics. Comput. Phys. Commun., 181(12), 2008-2023

Seddik H, Greve R, Zwinger T and Placidi L (2011) A full Stokes ice flow model for the vicinity of Dome Fuji, Antarctica, with induced anisotropy and fabric evolution. Cryosphere, 5(2), 495-508

Seroussi $\mathrm{H}$ and 6 others (2011) Ice flux divergence anomalies on 79north Glacier, Greenland. Geophys. Res. Lett., 38(9), L09501 (doi: 10.1029/2011GL047338)

Shao S and Lo EYM (2003) Incompressible SPH method for simulating Newtonian and non-Newtonian flows with a free surface. Adv. Water Resour., 26(7), 787-800

Tartakovsky AM and Meakin P (2005) Simulation of unsaturated flow in complex fractures using smoothed particle hydrodynamics. Vadose Zone J., 4(3), 848-855

Tartakovsky AM, Meakin P, Scheibe TD and Eichler West RM (2007) Simulations of reactive transport and precipitation with smoothed particle hydrodynamics. J. Comput. Phys., 222(2), 654-672

Tartakovsky AM, Tartakovsky DM, Scheibe TD and Meakin P (2008) Hybrid simulations of reaction-diffusion systems in porous media. SIAM J. Sci. Comput., 30(6), 2799-2816

Tartakovsky AM, Ferris KM and Meakin P (2009) Lagrangian particle model for multiphase flows. Comput. Phys. Commun., 180(10), 1874-1881

Weis M, Greve R and Hutter K (1999) Theory of shallow ice shelves. Contin. Mech. Thermodyn., 11(1), 15-50 